\title{
LA DISPUTA DEL GÉNERO EN EL ESTADO ESPAÑOL DESDE EL ANÁLISIS DEL ACTIVISMO CATÓLICO
}

\author{
Maribel Blázquez-Rodríguez*, Mónica Comejo-Valle* y José I. Pichardo-Galán
}

\section{Resumen}

A partir del desarrollo de las políticas de género en el Estado español, surgió la disputa en torno a la llamada «ideología de género» desde distintos actores antigénero, entre los que estaría la Iglesia Católica. La revisión de sus discursos, acciones y estrategias nos permite clarificar este término de «ideología» y, a su vez, dar cuenta de cómo hoy en día el género es un concepto político en disputa en nuestra sociedad, ya no tanto en el interior de la teoría feminista sino entre ciertos grupos contrarios al reconocimiento de los derechos de las mujeres y del colectivo LGTB. Para ello, también tomamos en cuenta la perspectiva de grupos católicos practicantes críticos que impulsan un mayor acercamiento entre la religión y los problemas sociales contemporáneos.

Palabras claves: Género, catolicismo, ideología de género, religión.

\section{Resumo}

A disputa do género no Estado espanhol a partir da análise do ativismo católico

O desenvolvimento das políticas de género pelo Estado espanhol fez com que diferentes atores sociais e políticos antigénero, como a Igreja Católica, encetassem uma disputa em torno da chamada "ideologia de género". A revisão dos seus discursos, ações e estratégias permite-nos clarificar o sentido do termo "ideologia" e, ao mesmo tempo, explicar como o género é hoje um conceito político em disputa na nossa sociedade, já não tanto no interior da teoria feminista, mas em certos grupos contrários ao reconhecimento dos direitos das mulheres e das pessoas LGBT. É também tida em conta a perspetiva de grupos católicos praticantes críticos que promovem uma relação mais próxima entre a religião e os problemas sociais contemporâneos.

Palavras-chave: Género, catolicismo, ideologia de género, religião.

\section{Abstract}

The gender dispute in the Spanish state from the analysis of the Catholic activism

Since the development of gender policies by the Spanish government, different social and political actors, including the Catholic Church, have been discussing the so-called "gender ideology». The revision of these anti-gender discourses, actions and strategies allows us to clarify the term «ideology» and, in turn, to explain how gender has become a political concept in dispute in our society, not only within feminist theory, but within cer-

\footnotetext{
* Docentes del Departamento de Antropología Social, Universidad Complutense de Madrid (UCM), Grupo de Investigación «Antropología, Convivencia y Diversidad» (GINADYC).

Dirección postal: Departamento de Antropología Social, Campus de Somosaguas, 28223 - Pozuelo de Alarcón (Madrid), España.

Correo electrónico: miblazquez@cps.ucm.es

Correo electrónico: mcornejo@cps.ucm.es

Correo electrónico: jipichardo@ucm.es
} 
tain groups opposed to the recognition of the rights of women and LGBT people. We also take into account the perspective of critical Catholic groups who promote a closer relationship between religion and contemporary social problems.

Keywords: Gender, Catholicism, gender ideology, religion.

\section{Introducción}

En este artículo, el objetivo es situar el debate entre género y religiones aportando datos y análisis de la experiencia en el contexto español. En este contexto ha sido clave la forma en que la perspectiva de género o las políticas de igualdad han sido incorporadas en la vida social y política, no sólo a través de diferentes regulaciones legislativas sino también apostando por un determinado desarrollo de la igualdad de género y los derechos sexuales y reproductivos. Aunque la batalla de algunos grupos religiosos contra las políticas de género se ha convertido en un fenómeno transnacional en los últimos años, España fue el primer país europeo en el que los activistas laicos católicos se movilizaron masivamente en las calles a partir de 2005 (Paternotte 2015), pero su discurso y su repertorio de movilización se extenderían hasta Argentina, en 2010, Francia, en 2011, Brasil, Polonia, Eslovaquia y Haití, en 2013, Italia y Hungría, en 2015, o Colombia, México, Chile, Bolivia, en 2016 (Bellé, Peroni, y Rapetti 2016; Cornejo-Valle y Pichardo-Galán, en prensa, 2017; Perintfalvi 2016). Los primeros pasos en España empezaron a darse cuando el parlamento legalizó el matrimonio entre personas del mismo sexo (2005). Desde entonces, distintos actores sociales se han manifestado públicamente tratando de revertir estas políticas de igualdad de género.

Para entender este proceso, se examinan varias cuestiones: primero, un breve acercamiento al desarrollo de la perspectiva de género en la sociedad española que ha conllevado las políticas de los derechos por la igualdad de las mujeres y los colectivos LGTB; surgiendo como reacción a estas políticas la adopción y difusión de la «ideología de género» protagonizada principalmente por actores religiosos - que se analiza en un segundo momento -, pero que se ha extendido al ámbito científico, como sucedió en la revista Arbor (2016). Junto con estas posiciones, se presentan las de algunos católicos que, desde una mirada crítica, plantean las relaciones entre el género con la religión católica, apostando por iniciativas más próximas a la teología feminista. Para concluir, se ofrece una reflexión que apuesta por la diversidad y la convivencia en medio de esta disputa entre el género y la religión en el Estado español.

\section{La conquista de la Igualdad en España y el uso de la categoría de «género»}

Pilar Folguera (2010) señala el recorrido particular del movimiento feminista en España, frente al de los países europeos y norteamericanos donde se desarrolló 
en los siglos XIX y XX, y aquí no será hasta bien entrado el siglo XX. La reclamación del derecho al voto puso de manifiesto dos corrientes dentro del feminismo: un feminismo conservador y confesional y el feminismo socialista y el anarquismo que consiguieron no sólo el voto en 1931 sino que las mujeres empezaran a disfrutar de sus derechos políticos junto a poder estudiar, trabajar, casarse civilmente, divorciarse, ejercer la patria potestad... No obstante, con el franquismo todos estos logros se invisibilizaron y se perdieron, teniendo que esperar a la aprobación de la Constitución de 1978 para la recuperación de la igualdad, que inició el proceso de modificación de distintas leyes discriminatorias. El movimiento feminista fue uno de los grandes impulsores de estas conquistas que ya claramente en 1979, en las I Jornadas Feministas celebradas en Granada, mostró nuevamente una división entre un feminismo de la igualdad centrado en los derechos y la igualdad y un feminismo de la diferencia centrado en las mujeres, como totalidad, como personas. No obstante, estas diferencias en el feminismo no han sido un impedimento para el desarrollo del feminismo institucional y académico que ha impulsado el principio de no discriminación, no sólo a nivel discursivo sino práctico, como se observa en la puesta en marcha de diversas legislaciones. Así entre sus logros, se encuentra, con el inicio del gobierno socialista, la Ley Orgánica 1/2004, de Medidas de Protección Integral contra la Violencia de Género, donde ya se hacía uso del término género referido a un sistema que organiza las relaciones sociales; posteriormente, la Ley 13/2005 reconoce el derecho a contraer matrimonio entre personas del mismo sexo. A continuación, la Ley orgánica de educación 2/2006 marcó desde su preámbulo que entre los fines de la educación se encuentra el reconocimiento de la diversidad afectivo-sexual. La principal polémica vendrá, no obstante, de la inclusión en el currículo oficial de la materia obligatoria «Educación para la ciudadanía y los derechos humanos», que señalaba explícitamente la necesidad de trabajar el rechazo a las discriminaciones por orientación afectivo-sexual y contra los prejuicios homófobos. Luego, la Ley 14/2006 sobre técnicas de reproducción humana asistida y la Ley $3 / 2007$, de Igualdad, donde se habla del principio de igualdad efectiva entre los hombres y las mujeres en diferentes ámbitos y de la perspectiva de género y se regula la rectificación registral de la mención relativa al sexo de las personas. En esta ley se institucionaliza por primera vez la perspectiva de género, que había ido desarrollándose por la teoría feminista y de acuerdo con las diferentes conceptualizaciones y declaraciones que se había hecho a nivel internacional, donde destaca la Cuarta Conferencia Mundial sobre la Mujer en Beijing en 1995. La apuesta por concretar la igualdad en la perspectiva de género quería llamar la atención sobre cómo las diferencias sexuales se convertían en desigualdades dentro de un sistema social (Díaz de Terán Velasco 2015) según distintos componentes (normas, roles, ideologías, poder...) (Scott 1986; Connell 1987; Lamas 1995). En consecuencia, ubicar el principio de igualdad en la perspectiva de género situaba la igualdad no sólo en las mujeres sino en el análisis de las relaciones entre los hombres y las mujeres, la sexualidad y el parentesco, quedando vinculados los derechos de las mujeres con los de los colectivos LGTB. 
Posteriormente, la Ley orgánica 2/2010, de salud sexual y reproductiva y de la interrupción voluntaria del embarazo, pretendía despenalizar el aborto y que fuera legal siempre que se lleve a cabo en las 14 primeras semanas de embarazo y sin intervención de terceras personas en la decisión. Además, permitía a las mujeres de 16 y 17 años abortar sin autorización de sus padres o tutores e incluía en su articulado el mandato a los poderes públicos del desarrollo de políticas sanitarias, educativas y sociales que garantizaban «la educación sanitaria integral y con perspectiva de género sobre salud sexual y salud reproductiva» y el mandato de que «la formación de profesionales de la salud se abordará con perspectiva de género».

Aunque todas estas leyes hacen referencia al desarrollo del género, sólo algunas de ellas levantaron resistencias, haciendo emerger los discursos acerca de la «ideología de género», sin duda las que cuestionaban los modelos de familia y los derechos sexuales y reproductivos. Así, la «ideología de género» proporciona coherencia entre dos preguntas ante las que la Iglesia tiene una larga historia de lucha: la de las mujeres y la de la orientación sexual (Bracke and Paternotte 2016).

Sin embargo, a partir del 2011, año de entrada en el gobierno del Partido Popular, se ha ido eliminando la palabra «género» de documentos oficiales, textos legales, convocatorias de proyectos, políticas públicas, etc. y se ha sustituido por otros términos como «mujeres» o «igualdad» (Cornejo-Valle y Pichardo-Galán 2017).

\section{El surgimiento de la «ideología de género» en el Estado español}

Para ubicar el origen del término «ideología de género» hay que situarse en las conferencias de El Cairo (1994) y Beijing (1995) en las que se inicia el uso institucional y político de la categoría género y la lucha por los derechos de las mujeres, entre los que destaca la enunciación por primera vez de los derechos sexuales y reproductivos (Cornejo-Valle y Pichardo-Galán 2017). Como se relata en la crónica de Doris E. Buss (1998), en 1994, la reunión de El Cairo sobre población sirvió para orquestar una oposición creciente entre ciertos grupos católicos y musulmanes contra posibles acuerdos internacionales en cuestiones relacionadas con la familia. Como estrategia, el Vaticano obstaculizó constantemente las discusiones y los acuerdos al negarse a aceptar conceptos como género o «familias» en plural. Así en El Cairo, Dale O'Leary difunde un informe «Gender, the deconstruction of women» que será la semilla del libro que publica posteriormente, The gender agenda. Redefining equality (O'Leary 1997). En 1998, Monseñor Alzamora Revoredo, entonces presidente de la Comisión ad hoc de la Mujer de la Conferencia Episcopal Peruana, publica el primer texto de esta tendencia en español bajo el título La Ideología de Género. Sus Peligros y Alcances (que es el mismo texto que aparece traducido en varios idiomas en el Lexicón de 2003).

Judith Butler (2017) plantea que, siguiendo el trabajo de Richard Miskolci y Maximiliano Campana, Joseph Ratzinger en 1997, antes de convertirse en el Papa 
Benedicto, formula esta idea del género como ideología. Conviene también apuntar el trabajo de Mary Anne Case (2016) quien muestra cómo el discurso sobre las relaciones entre las mujeres y los hombres, la perspectiva de la complementariedad basada en la relación «natural» y biológica es una innovación reciente formulada por los últimos Papas (Pío XII, Juan Pablo II y Benedicto XVI).

Sarah Bracke y David Patternotte (2016) insisten en la relevancia de analizar cuáles han sido las estrategias propias de esta cruzada antigénero en cada uno de los contextos que, aunque con elementos comunes, dispone de un desarrollo particular según cada contexto; en su artículo analizan los discursos del cardenal Robert Sarah. Por tanto, conviene no dejar en el olvido a otros autores que han sido cruciales en la creación, difusión y promoción de esta ideología de género, además de O'Leary destacan, en Latinoamérica, Jorge Scala (Vaggione 2016) y Christine de Marcellus de Vollmer; en Europa, Lucetta Scaraffia y Gabrielle Kuby (Perintfalvi 2016) y, en Estados Unidos, Mary Ann Glendon (Vaggione 2016). Pues bien, en la Iglesia Católica española aparece esta expresión en una instrucción pastoral de la Conferencia Episcopal Española (CEE) titulada La familia, santuario de la vida y esperanza de la sociedad (CEE 2001), en la que se denunciaba el alineamiento de una parte de la sociedad española con una línea política que procede de «foros internacionales» y que pretende introducir

toda una serie de «nuevos derechos» que, en el fondo, no son más que la pretensión de una «libertad sexual» sin limites: derecho a la anticoncepción, a la salud reproductiva, al libre diseño de la sexualidad, a la elección del «modelo de familia», a la institucionalización de las uniones homosexuales, etc. (CEE 2001, 34).

Habría dos estrategias que se desarrollan conjuntamente y que conviene situar: en primer lugar, se opta por usar la expresión «ideología» en su sentido marxista como una estrategia consciente de la influencia del lenguaje en la formación de la opinión pública, a fin de denunciar el carácter ilusorio del término "género», en cuanto a construcción social. En este sentido, a la perspectiva de género, renombrada como «ideología de género», se le atribuye la perversión interpretativa de promover los derechos humanos como herramientas para las reivindicaciones de las mujeres y de las minorías sexuales jugando a la confusión terminológica. Este juego de distorsión semántica intenta menoscabar las connotaciones positivas del término "género» (asociadas a la igualdad entre hombres y mujeres como valor) al tiempo que impone nuevas connotaciones negativas: asociándolo al concepto de «ideología» que equiparan a «falsedad» (frente a su discurso «verdadero») e identificando el término «género» hacia lo que la Iglesia Católica llama «una cultura de la muerte».

Bracke y Patternotte $(2016,144)$ también sugieren que hablar de ideología es un ejercicio retórico particular que asocia la producción de género al mundo de las creencias, socavando con este proyecto y, a la par, se producen conocimientos procedentes de la biología o la medicina que enfatizan el sentido común y natural del 
sexo en contra del género. Asimismo, Butler reclama también que el género no es una ideología sino la expresión de un derecho y libertad básico, utilizar esta denominación es situarlo en: «Un punto de vista ilusorio y dogmático, que se ha `apoderado' del pensamiento de las personas de una manera acrítica» $(2017,6)$.

En segundo lugar, a este desplazamiento semántico lo acompaña una segunda estrategia clave: plantear el desorden de los valores como resultado de los avances en el reconocimiento de los derechos sexuales y contra el feminismo y el movimiento LGBT, a fin de activar un cierto «pánico moral» (Herdt 2009). Como sostienen Teresa Toldy y Ana Cristina Santos (2016), los documentos del Vaticano y las Conferencias Episcopales en diversos países hablan de «ideología de género» porque sostener la idea que propone el género como un sistema construido socioculturalmente implica desvirtuar el plan original para los sexos, que es natural, atemporal y no puede ser cuestionado, pues procede de lo trascendente. Estas autoras inciden en que aludir al concepto de la naturaleza se convierte en una forma de mantener el orden social. Al respecto, conviene recuperar a Verena Stolcke que mencionaba la «naturalización» como

el subterfugio ideológico en la sociedad de clases para reconciliar la ilusión liberal de que todos los seres humanos, libres e iguales por nacimiento, gozan de igualdad de valor y oportunidades, con la desigualdad socioeconómica existente, en interés de los que se benefician de ésta última (Stolcke 1992, 29).

En este sentido, aquí sería utilizado para impedir nuevas formas de pensar, vivir y finalmente de construir la ciudadanía.

En otros artículos (Cornejo-Valle y Pichardo-Galán 2017), hemos referido que, como las posiciones católicas no tuvieran el éxito deseado en las conferencias internacionales, se puso en marcha una acción más planificada. A partir de la segunda mitad de la década de 1990, la respuesta proactiva contra los avances de género se basaría en la declaración de una alerta mundial contra los avances en los derechos sexuales y reproductivos, con dos dimensiones: una intelectual claramente internacional y otra política, con elementos internacionales y locales. Por lo que respecta a la primera, el trabajo intelectual empezó con la investigación a fondo de la literatura sobre género concebida en el feminismo crítico y continuó con la difusión de una interpretación católica de los derechos humanos, beligerante contra el feminismo, el marxismo y su síntesis en la llamada «ideología de género». La segunda dimensión, de carácter político, se caracterizaba por una activa oposición a todas las acciones relacionadas con la igualdad.

En el Estado español, hemos planteado que habría cuatro momentos claves o cuatro olas de movilización contra el género (2004-2005, 2006-2009, 2010-2012 y 2015) relacionadas con la aprobación de ciertas legislaciones (Cornejo-Valle y Pichardo-Galán 2017). El primer momento se inicia al ganar las elecciones el PSOE, con el reconocimiento del matrimonio entre parejas del mismo sexo que se regula en 2005. Un segundo momento, considerado el que inaugura este discurso por 
parte de la Iglesia Católica española y, a partir de ahí, es asumido por los activistas laicos. En 2007, se inicia una movilización de objeción contra la materia de «Educación para la ciudadanía» dirigida a los padres pues se entiende como una amenaza a la libertad religiosa, pero tuvo una escasa objeción y el Tribunal Supremo español consideró que la objeción de conciencia no podía ser aplicada a esta materia (Cornejo-Valle y Pichardo-Galán 2017). El tercer momento, que produce una fuerte respuesta, tiene que ver con la ley de interrupción voluntaria del embarazo (2010). El cuarto y último momento se inicia con las elecciones de 2015 y la manutención del Partido Popular en el gobierno, ahora sin mayoría absoluta y, por eso, sin el estilo de confrontación característico de la época anterior, pero en el que se hace más patente el esfuerzo enmarcador de los actores religiosos, los cuales abordaremos en el siguiente apartado.

\section{Los actores y estrategias contra el género en el Estado español}

En Cornejo-Valle y Pichardo-Galán (en prensa) se desarrolla más ampliamente quiénes son estos actores, aquí simplemente serán referidos algunos datos para situar quiénes son los principales activistas antigénero en el panorama español: (1) la jerarquía de la Iglesia Católica, con diferentes obispos y cardenales que no sólo firmaron y publicaron documentos doctrinales sino que también participaron en manifestaciones, promovieron misas en masa en defensa del modelo católico de familia como «familia natural». (2) Las asociaciones contrarias al aborto tradicionales, donde destaca el Foro Español de la Familia, formado por diferentes asociaciones, mayoritariamente católicas. (3) Un nuevo lobby neoconservador agrupado en la organización Hazteoir.Org, que desde el 2005 ha participado activamente conjuntamente con el Foro de la Familia, pero también a nivel independiente con una plataforma web y un activismo más internacional. Una de sus últimas acciones, lanzada en febrero de 2017, fue una campaña a través de un autobús que denominaron «el bus de la libertad» con lemas tránsfobos: «Los niños tienen pene, las niñas tienen vulva. Que no te engañen» y «Si naces hombre, eres hombre. Si eres mujer, seguirás siéndolo»; y se desplazó en el centro de la ciudad de Madrid, y han puesto en circulación en otras ciudades del mundo como Nueva York. Y (4), los partidos de extrema derecha, que se han puesto de manifiesto recientemente, a partir de 2014.

Estos cuatro grupos de actores difieren en su composición social, en su naturaleza, en su posición política y en el uso de recursos y estrategias de movilización, pero todos comparten una agenda afín en su pelea contra: el matrimonio y la adopción para parejas del mismo sexo, la interrupción voluntaria del embarazo, la educación sexual y de género, la reproducción asistida, la gestación subrogada, la violencia de género, las problemáticas transgénero y transexuales (especialmente respecto al acceso a los servicios públicos de salud) e, incluso, contra la presencia de estudios de género en las universidades públicas. 
A estos actores, queremos incorporar aquellos que han participado en iniciativas académicas como el I Congreso Internacional de Ideología de Género en la Universidad de Navarra, dirigida por el Opus Dei, ${ }^{1}$ en el que la mayor parte de participantes del programa pertenecen a universidades católicas y que no tuvo impacto mediático ni académico significativo (Cornejo-Valle y Pichardo-Galán 2017). Sin detenernos en otros eventos desarrollados en universidades católicas sí quisiéramos presentar el caso que se da en el ámbito científico a partir de la publicación del monográfico número 778 de la revista Arbor, del Consejo Superior de Investigaciones Científicas (CSIC), titulado: ¿Hay mujeres más allá del feminismo? De la lucha por la igualdad de género al tranhumanismo/posthumanismo. En sus once artículos, se defiende que la igualdad ya está conseguida y, por tanto, ya no es preciso el feminismo ni la «ideología de género», término bajo el que se vuelve a hablar del género. La directora del monográfico, la catedrática sevillana María Caballero Wangüemert señala:

A partir de los setenta, comienza a difundirse la ideología de género, hoy omnipresente en el discurso antropológico, social, legal y político. Un nuevo paradigma que disuelve la tradicional imagen del ser humano en cuanto persona, como unidad sexuada (cuerpo y espíritu) que en la Europa cristiana tuvo su aval en el doble relato de la creación del Génesis: varón y mujer serían dos modos distintos y complementarios de encarnar ese «ser persona». Así lo ha recordado Juan Pablo II en sus homilías sobre la teología del cuerpo (1995) y en su Carta a las mujeres: «Femineidad y masculinidad son entre sí complementarias no sólo desde el punto de vista físico y psíquico, sino ontológico. Solo gracias a la dualidad de lo masculino y de lo femenino, lo humano se realiza plenamente» (1996, p. 38). E incluso - una afirmación fuerte- en cada unión conyugal «se renueva, en cierto modo, el misterio de la creación en toda su profundidad originaria y fuerza vital» (1995, p. 81). Ello supone que la masculinidad o feminidad se extiende a todos los ámbitos de su ser: algo estudiado por la ciencia en libros como Cerebro de mujer, cerebro de varón, de López Moratalla (Caballero Wangüemert 2016, 2).

En este ejemplo, se observa cómo se desmantela el género, se defiende la complementariedad basada en la diferencia sexual como algo biológico y tradicional, que se enraíza en la Biblia y en discursos papales. Junto a ello, otra vez se emplea la estrategia del pánico y el miedo: «De modo que se fragmenta, cae rota en pedazos esa imagen armónica en que ambos aspectos al unísono conforman su identidad masculina o femenina» (Caballero Wangüemert 2016, 2).

Otro de los artículos corresponde a la profesora de filosofía de la Universidad de Navarra, Lourdes Flamarique, vinculada con el grupo católico Opus Dei, quien propone una nueva mirada de la modernidad como «una perspectiva integradora o el más acá de los estudios de género» (2016). En su texto afirma que la «ideología de género»: "pretende erradicar la distinción entre lo femenino y lo masculino y, con ello, las desigualdades conocidas» (Flamarique 2016, 5). Considera que el

$<$ http://www.unav.edu/congreso/ideologiadegenero $>$. 
énfasis en la eliminación de las diferencias para superar la dominación es utópico pues hay más diferencias que las derivadas del género y, además, la diferencia constituye diversidad. En este sentido descontextualizado también aparece la afirmación de la profesora Ángela Aparisi Miralles, quien defiende el modelo de la igualdad en la diferencia, que denomina de la reciprocidad, complementariedad o corresponsabilidad como aquel que: «permite una armónica combinación entre dichos principios, lo cual lo sitúa en un mejor punto de partida para el debate en el ámbito de los derechos humanos» (Aparisi Miralles 2016, 1). En estas interpretaciones no se considera las discriminaciones, desigualdades y violencias que en los diferentes ámbitos (laboral, educativo, político, económico...) hoy siguen sufriendo más las mujeres y las minorías sexuales en nuestra sociedad.

Ante estas aseveraciones en una revista científica, sostenida por fondos públicos, respondieron la Asociación de Mujeres Investigadoras y Tecnólogas (AMIT), mediante una recogida de firmas y la publicación de una carta de la que también se hicieron eco algunas revistas ${ }^{2}$. En la carta se solicitaba la retirada de este número pues reflejaba ideologías religiosas y no científicas, así como se devaluaba el feminismo sin una argumentación basada en investigaciones o en casos. Hubo distintas respuestas en contra en algunos medios de comunicación, que apoyaban el monográfico y razonaban, primero, el carácter científico de la perspectiva de las autoras; segundo, que el género era la imposición de una teoría, denominándola «totalitarismo», y, tercero, justificando que la mirada de género es «anti-natural» ${ }^{3}$. El director de la revista respondió indicando que: «En la actividad científica en cuestión de ciencias humanas hay diferentes escuelas y modos de aproximarse a la realidad, y eso es enriquecedor y nunca excluyente», ${ }^{4}$ de ahí el encargo de hacer este monográfico a estas autoras.

\section{Respuestas críticas desde el catolicismo}

Para comprender las reacciones, apoyos y alcance de estos grupos y discursos antigénero debemos considerar cómo es la adscripción religiosa en nuestro país. Aunque España sea un país donde la mayoría de la población se identifica como católica (más del 70\%), los católicos practicantes representan sólo una minoría: un 14-15\% bien porque asiste a ceremonias religiosas de forma regular o se considera así (Cornejo-Valle y Pichardo-Galán 2017). Igualmente, sólo el 3\% de los españoles considera la religión como uno de los principales valores en su vida (la media

\footnotetext{
$2 \quad<$ http://www.eldiario.es/sociedad/revista-CSIC-cuestiona-discriminacion-cristal_0_558194677. html $>$.

$3 \quad<$ https://www.religionconfidencial.com/tribunas/ideologia-genero-revista-ARBOR_0_2808319166.html>.

$4 \quad<$ https://www.eldiario.es/sociedad/revista-CSIC-cuestiona-discriminacion-cristal_0_558194677. html $>$.
} 
europea es del 5\%). Es decir, que la autodefinición como persona católica parece entonces tener más que ver con una identidad cultural (se es católico porque no se es musulmán, ni judío, ni budista...) que con una creencia religiosa fuertemente arraigada en la persona (Cornejo-Valle, Pichardo-Galán y Henríquez 2013).

Si bien hay un $10 \%$ de católicos españoles que están alineados con la jerarquía eclesiástica contra los derechos sexuales y reproductivos, al mismo tiempo, la mayoría de españoles y españolas que se definen como católicos no comparten las mismas ideas que sus obispos y la mayor parte se muestra a favor del matrimonio entre personas del mismo sexo $(63 \%)$, a favor del uso de anticonceptivos $(90 \%)$, del divorcio (82\%), del sacerdocio de las mujeres $(78 \%)$ y del aborto (88\% a favor: $24 \%$ siempre y 64\% en algunos casos) (Cornejo-Valle y Pichardo-Galán 2017).

E incluso entre los católicos practicantes encontramos posiciones dispares respecto a estas cuestiones, como hemos podido constatar en una investigación sobre expresiones religiosas en el espacio público, ${ }^{5}$ en la que estamos realizando entrevistas a católicos quienes manifiestan su discrepancia con este tipo de acciones que consideran que incrementa la distancia entre la Iglesia, como institución, y los creyentes, reclamando una implicación más firme con los problemas sociales actuales, como la igualdad entre los hombres y las mujeres. Así, se hace un análisis más diacrónico de los propios cambios dentro de las instituciones eclesiales y estos se relacionan más con el talante de los obispos o papas:

La nueva línea eclesial evita actos masivos como la Misa de la Familia que convocaba Monseñor Rouco y que representaba el sector eclesial más beligerante. Con la llegada de Monseñor Osoro el talante es más acorde con el del Papa Francisco. Desde las instancias oficiales no se ha apoyado el gesto del autobús de Hazte oír y la campaña se disuelve, con poco eco en la/os católicos de Madrid (Entrevista a monja teóloga, marzo 20176).

Respecto a esta campaña también otras entrevistadas se sienten incómodas porque transmiten un mensaje de segregación:

Me llego el mensaje de «los niños tienen pene, las niñas tienen vagina» y no me parece bien, no me gusta que lo digan, no hay que decir, o sea, tenemos que educar en la aceptación de las diferentes, entonces no me gustaba ese autobús, el mensaje no me gustaba, no lo tenía muy claro, pero no me gusta (Entrevista a teóloga, laica, casada con hijos, marzo 2017).

5 Proyecto: «Expresiones religiosas en el espacio urbano en Madrid y Barcelona. Un estudio de casos múltiple sobre la visibilidad de la diversidad religiosa en el espacio público». Referencia: CSO2015-66198-P. Convocatoria Pública de Ayudas correspondientes al Programa Estatal de Investigación, Desarrollo e Innovación Orientada a los Retos de la Sociedad, en el marco del Plan Estatal de Investigación Científica y Técnica y de Innovación 2013-2016. Investigadora principal: María del Mar Griera, grupo de investigación ISOR, de la Universidad Autónoma de Barcelona. Por razones éticas propias de la investigación social y bajo petición de algunos informantes, los autores de los discursos recogidos en entrevistas se han anonimizado. 
Igualmente, al plantear el papel de las mujeres en la Iglesia se recoge toda la historia androcéntrica y misógina que no es ajena al catolicismo y sigue presente, y sus propuestas se centran en la incorporación de las mujeres:

La Iglesia tiene por delante la tarea de promover la plena participación de las mujeres en ella, su compromiso por la justicia, la paz y la salvaguarda de la creación, su participación en la teología, la liturgia, la catequesis y la comunicación espiritual. Todo lo que elabore sin la experiencia, la reflexión, la sensibilidad de las mujeres está necesitando una revisión, una compleción. Y sin ello la Iglesia no podrá ser verdaderamente la Iglesia que quiso Jesús (Entrevista a monja teóloga, marzo 2017).

Como explicaciones para entender esta falta de apoyo a los actores antigénero, por una parte, sería porque ha sido una estrategia de confrontación que ha puesto de manifiesto la separación entre una minoría de radicales y una mayoría moderada (Cornejo-Valle y Pichardo-Galán 2017) dentro tanto del catolicismo practicante como de la sociedad española. Y, por otra parte, podría ser explicada porque, como afirmaban nuestros informantes, una parte incluso de las personas católicas están más a favor de la igualdad y la incorporación de los logros sociales también en el interior de las religiones. Lo cual no excluye otras explicaciones como la opción del apoyo a las hijas e hijos gays y lesbianas frente a los mandatos de la Iglesia o la tradición cultural de anticlericalismo popular bien asentada en la historia española (Cornejo-Valle y Pichardo-Galán 2017).

\section{Conclusiones}

Hemos titulado este artículo «la disputa de género» queriendo hacernos eco del escarnio sufrido por la teórica Butler recientemente en Brasil ${ }^{7}$, justificado porque difundía la «ideología de género». Butler en 1989 publico el texto «El género en disputa» donde como ella misma expresa:

Buscaba afirmar la complejidad de nuestras identificaciones de género y deseos y unirse a aquellos que dentro del movimiento LGBTQ contemporáneo creían que una de las libertades fundamentales que deberían respetarse es la libertad de expresión de género. ¿Era ésta una negación de la existencia de las diferencias naturales entre los sexos? Ciertamente nunca lo fue (Butler 2017, 1).

Los actores antigénero han desarrollado diferentes estrategias para frenar las políticas de igualdad de género: la utilización del término «ideología de género» para distorsionar esta categoría, la estigmatización junto con activar y promover el

\footnotetext{
$<$ https://elsaltodiario.com/brasil/ultraconservadores-firmas-judith-butler-brasil-insultos>.
} 
pánico moral y una «nueva evangelización» de España. ${ }^{8}$ Aunque, como aseveran Brake y Paternotte (2016), el rechazo a la «ideología de género» no niega, sino que irónicamente reafirma los vínculos conceptuales del género con el sexo, sexualidad, reproducción y familia, y, por tanto, podríamos definirlo como un signo de la potencialidad y competencia tanto teórica como práctica de la categoría de género. La realidad es que la incorporación del género se está convirtiendo en una batalla.

La «ideología de género» se está presentando como la causa y el causante de numerosos y diversos males de nuestra sociedad, se le otorga la responsabilidad de las prácticas abortivas, rupturas matrimoniales, esterilizaciones, descenso de la nupcialidad y retraso de la edad del matrimonio, violencia en la convivencia doméstica y adicciones a la pornografía, las drogas, el alcohol, el juego o internet (Cornejo-Valle y Pichardo-Galán, en prensa), como si estos males hubieran sido creados por los movimientos feministas y LGTB. Bajo estas acusaciones no sólo se excluye un análisis de la complejidad de cada uno de estos procesos y los factores intervinientes, sino que se inicia una estigmatización junto a un anatema (CornejoValle y Pichardo-Galán, en prensa) y una persecución tanto de los discursos como de los actores sociales, como apropiadamente indica Butler (2017) en respuesta al ataque recibido, se inicia una caza de brujas, continuista con la realizada por la Iglesia en otros momentos históricos, dirigida a aquellas personas y creencias que no encajan con sus dogmas.

Reconociendo en el Estado español los logros alcanzados en los último 12 años por las políticas de género, convendría subrayar que las controversias han aparecido cuando se ha tocado ciertos ámbitos sociales como la sexualidad, la reproducción y la familia, mientras que cuando venía a corregir una situación histórica de desigualdad como la que se daba en nuestro país no han surgido estas voces discordantes, ante ello no habría que dar por supuesto a qué se deben estas reacciones: ¿qué elementos del orden social están siendo cuestionados? ¿qué relaciones de poder están siendo invertidas? Por una parte, se está poniendo en juego la reproducción biológica y del orden social, de un orden patriarcal (Blázquez-Rodríguez y Montes-Muñoz 2012), que pone en marcha todos los mecanismos posibles para impedir el cambio (desde el uso del término «ideología», naturalización, estigmatización, persecución, pánico y evangelización). Por otra parte, se está poniendo en el mismo nivel de equiparación las elecciones y vidas de aquellas personas que salen de los modelos heteronormativos, que están ganando el reconocimiento y el prestigio social de otras formas de vida. Obviamente esta igualación pone en marcha todos los medios patriarcales de dominación que van desde la creación de representaciones y discursos como si fueran unívocos, e implican el control de las conciencias hasta formas de control de los cuerpos y las vidas de las personas y estas formas de dominación tienen que ver con el uso del poder, tanto el coercitivo, usándose la violencia tanto física como a través de sanciones y castigos, como el poder simbólico para imponer y disuadir el cambio.

\footnotetext{
<http://www.conferenciaepiscopal.es/images/stories/documentos/2012PlanPastoral2011-2015.pdf>.
} 
Hoy, como en otros momentos históricos, la vindicación por el género sigue siendo no solo un asunto crucial sino una disputa que va más allá del uso del concepto de género, pues busca a quién responsabilizar de ciertos cambios sociales contemporáneos y detrás de todo ello, lo que se está poniendo en juego es la libertad. Por parte de estos grupos se indica que las políticas de igualdad y el género restringen la libertad de los católicos para practicar y expresar su fe; y quienes apoyan la igualdad de género defienden el derecho a decidir sobre sus cuerpos, autonomía, movilidad, en fin, el derecho a una vida libre, digna y reconocida. Por lo que asistimos a un tiempo en el que, como en otros momentos históricos, este término se convierte en un elemento en disputa entre quienes quieren suprimirlo y quienes quieren seguir utilizándolo, con la novedad del cambio entre los actores de esta disputa, no son las diversas corrientes feministas sino entre partidarios y opositores al feminismo. Por tanto, convendría que el feminismo no olvidara estas disputas y no se detuviera mucho tiempo en un movimiento centrípeto sobre las divergencias y problemáticas del término género y entre las distintas corrientes como el movimiento LGTB (Moreno y Pichardo-Galán, 2006), que desviarán su atención acerca del carácter político que hoy, en contextos como el español, adquiere su uso.

Hoy, aquí, en el Estado español, la categoría de género ha cumplido con el trabajo político que se esperaba -poner de manifiesto el orden social heteronormativo y androcéntrico histórico y construido (Butler, Scott, y Fassin, 2007) y desafiar a su cambio. Nuevamente las religiones, aquí el catolicismo, desempeñan un papel fundamental como instituciones reproductoras del orden social, tratando no sólo de mantener una representación propia de las relaciones entre los hombres y las mujeres en el interior de su confesión sino de proponerla como la única, la histórica y la natural. Sin embargo, como lo señala un número importante de estudios, las religiones tienen posturas dispares respecto de la sexualidad y la reproducción y parte de esta disparidad obedece al impacto de los movimientos feministas y por la diversidad sexual así como a visiones críticas dentro del catolicismo, que dentro de esa minoría de católicos practicantes, sugieren posturas tanto de la Iglesia como entre los creyentes que tengan más en cuenta los cambios sociales actuales, como muestran las personas católicas entrevistadas. Sus discursos son apuestas, como este artículo, por la convivencia y la lucha por la democracia, en la que seguiremos teniendo que debatir sobre el género pero con el género.

\section{Referencias bibliográficas}

Aparisi Miralles, Angela. 2016. «Discursos de género: el modelo de la igualdad en la diferencia». Arbor 192 (778): a303. DOI: https://doi.org/10.3989/arbor.2016.778n2006

Blázquez-Rodríguez, Maribel, y María Jesús Montes-Muñoz. 2012. «La reproducción en la vida de las mujeres». Revista Mètode 76: 65-69. https://metode.org/issues/monographs/ reproduction-in-womens-lives.html 
Bellé, Elisa, Caterina Peroni, y Elisa Rapetti. 2016. «Fighting about (Sexual) Citizenship: Italy's 'Nature or Culture' Dilemma», Revista Crítica de Ciências Sociais 110: 73-98. DOI: https://doi.org/10.4000/rccs.6379

Bracke, Sarah, y David Paternotte. 2016. «Unpacking the Sin of Gender». Religion \& Gender 6 (2): 143-154. DOI: https://doi.org/10.18352/rg.10167

Buss, Doris E. 1998. «Robes, relics and rights: the Vatican and the Beijing Conference on women». Social \& Legal Studies 7(3): 339-363. DOI: http://journals.sagepub.com/doi/ pdf/10.1177/096466399800700302

Butler, Judith. 2017. «Derecho a réplica. El fantasma del género». Página 12. Disponible en https://www.pagina12.com.ar/77673-el-fantasma-del-genero

Butler, Judith, Joan Wallach Scott, y Éric Fassin. 2007. «Pour ne pas en finis avec le «genre»... Table ronde». Sociétés E Représentations 2 (24): 285-306. DOI: <https://dokodoc.com/ pour-ne-pas-en-finir-avec-le-genre-table-ronde.html>.

Caballero Wangüemert, María. 2016. «¿Hay mujeres más allá del feminismo? De la lucha por la igualdad al transhumanismo/posthumanismo». Arbor 192 (778): a297.

Case, Mary Anne. 2016. «The Role of the Popes in the Invention of Complementarity and the Vatican's Anathematization of Gender». Religion \& Gender 6 (2): 155-172. DOI: https://doi.org/10.18352/rg.10124

CEE - Conferencia Episcopal Española. 2001. La familia, santuario de la vida y esperanza de la sociedad. Madrid: CEE. Disponible en http://www.conferenciaepiscopal.es/documentos/Conferencia/familia_santuario2.htm\#2

Connell, Raewyn. 1987. Gender and Power: Society, the Person and Sexual Politics. Stanford, CA: Stanford University Press.

Cornejo-Valle, Mónica, y José Ignacio Pichardo-Galán. 2017. «La 'ideología de género' frente a los derechos sexuales y reproductivos. El escenario español». Cadernos Pagu 50. DOI: https://doi.org/10.1590/18094449201700500009

Cornejo-Valle, Mónica, y José Ignacio Pichardo-Galán. En prensa. «Actores y estrategias en la movilización anti-género en España: el desplazamiento de una política de Iglesia al activismo laico». Revista de psicología política.

Cornejo-Valle, Mónica, José Ignacio Pichardo-Galán, y Andrea Henríquez. 2013. «Homosexualidad y religión ¿derechos irreconciliables?» En Actas del XI Congreso Español de Sociología (Vol. III), Madrid, Federación Española de Sociología, 563-573.

Díaz de Terán Velasco, y Maricruz. 2015. «La transversalidad de género. Valoraciones a partir de algunos documentos de las Naciones Unidas». Revista Anuario Español de Derecho Internacional 31: 323-347. DOI: https://doi.org/10.15581/010.31

Folguera, Pilar. 2010. El feminismo en España: dos siglos de historia. Madrid: Editorial Pablo Iglesias.

Flamarique, Lourdes. 2016. «Modernidad y cambio social: una perspectiva integradora, o el más acá de los estudios de género». Arbor 192 (778): a301. DOI: https://doi.org/10.3989/ arbor.2016.778n2004

Lamas, Marta. 1995. El género: la construcción cultural de la diferencia sexual. México, DF: UNAM.

Herdt, Gilbert, ed. 2009. Moral Panics, Sex Panics: Fear and the Fight over Sexual Rights. New York: New York University Press.

Moreno Sánchez, Ángel, y José Ignacio Pichardo-Galán. 2006. «Homonormatividad y existencia sexual. Amistades peligrosas entre género y sexualidad». AIBR. Revista de Antropología Iberoamericana 1 (1): 143-156. DOI: https://doi.org/10.11156/aibr.010110

O'Leary, Dale. 1997. The gender agenda. Redefining equality. Lafayette, LA: Vital Issues Press.

Paternotte, David. 2015. «Blessing the Crowds. Catholic Mobilizations against Gender in 
Europe». En Anti-Genderismus. Sexualität und Geschlecht als Schauplätze aktueller politischer Auseinandersetzungen, editado por S. Hark y P. I. Villa. Bielefeld: Transcript Verlag. Perintfalvi, Rita. 2016. «The True Face of the 'Gender Ideology' Discourse. Religious Fundamentalism, or Questioning the Principle of Democracy?» Journal of the European Society of Women in Theological Research 24: 47-62. DOI: https://doi.org/10.2143/ ESWTR.24.0.3170025

Scott, Joan. 1986. «Gender: A Useful Category of Historical Analysis». The American Historical Review 91 (5): 1053-1075. DOI: https://doi.org/10.2307/1864376

Stolcke, Verena. 1992. «¿Es el sexo para el género como la raza para la etnicidad?» Mientras tanto 48: 87-112.

Toldy, Teresa Martinho, y Ana Cristina Santos. 2016. «Religião, género e cidadania sexual: Uma introdução». Revista Crítica de Ciências Sociais 110: 43-50. DOI: http://journals. openedition.org/rccs/6370

Vaggione, Juan Marco. 2016. «Francis and 'Gender Ideology': Heritage, Displacement and Continuities». Religion \& Gender 6 (2): 302-307. DOI: https://doi.org/10.18352/rg.10184

Maribel Blázquez Rodríguez. Profesora Contratada Doctora en la Universidad Complutense de Madrid. Doctora en Antropología por la Universidad Rovira i Virgili de Tarragona. Licenciada en esta disciplina por la Universidad Autónoma de Madrid. Primer Premio Victoria Kent (XX Edición) del Seminario de Estudios Interdisciplinarios de la Mujer de la Universidad de Málaga. Ha impartido clases en el Máster Oficial en el Programa de Formación de la Especialidad de Enfermería Obstétrico-ginecológicas (Matronas) de la Universidad Autónoma de Madrid y en el Instituto de Salud Carlos III. Correo electrónico: miblazquez@cps.ucm.es

Mónica Cornejo-Valle. Profesora Contratada Doctora en la Universidad Complutense de Madrid, co-directora del Grupo de Investigación Antropología, Diversidad y Convivencia. Doctora en Antropología por la Universidad Complutense de Madrid. Licenciada en Filosofía y en Antropología Social por la Universidad de Deusto. XVI Premio Nacional de Investigación Cultural Marques de Lozoya (2007). Ha sido profesora en la Universidad de Castilla-La Mancha y en el Instituto Social León XIII (Salamanca). Visiting scholar en la Universidad de Cambridge y Universita degli Studi di Siena.

Correo electrónico: mcornejo@cps.ucm.es

José Ignacio Pichardo-Galán. Profesor Contratado Doctor en la Universidad Complutense de Madrid. Doctor en Antropología Social por la Universidad Autónoma de Madrid. Vicedecano de Estudiantes en la Facultad de Trabajo Social y ha sido coordinador de la Oficina de Diversidad Sexual e Identidad de Género UCM. Ha dado clase y/o investigado en la Universidad de Barcelona, San Francisco State University, Laboratoire de Sciences Sociales de Paris, Université du Québec à Montréal, Universidade Estadual Paulista, Universidad de Buenos Aires y Western Sydney University. También ha sido profesor en Syracuse University Madrid y Visiting Professor en Università degli Studi di Foggia.

Correo electrónico: jipichardo@ucm.es

Artículo recibido el 30 de noviembre de 2017 y aceptado para publicación el 2 de marzo de 2018. 